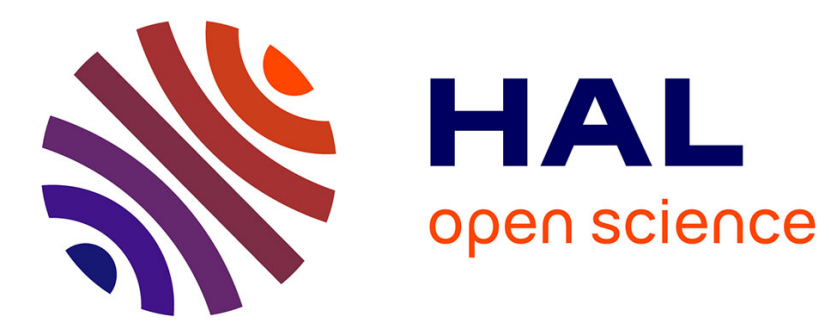

\title{
La recherche empirique en histoire à l'ère numérique
}

Nicole Dufournaud

\section{To cite this version:}

Nicole Dufournaud. La recherche empirique en histoire à l'ère numérique . La Gazette des Archives , 2015, Voyages extraordinairement numériques : 10 ans d'archivage électronique, et demain ?, 2015-4 (240). hal-01307117

\section{HAL Id: hal-01307117 https://hal.science/hal-01307117}

Submitted on 26 Apr 2016

HAL is a multi-disciplinary open access archive for the deposit and dissemination of scientific research documents, whether they are published or not. The documents may come from teaching and research institutions in France or abroad, or from public or private research centers.
L'archive ouverte pluridisciplinaire HAL, est destinée au dépôt et à la diffusion de documents scientifiques de niveau recherche, publiés ou non, émanant des établissements d'enseignement et de recherche français ou étrangers, des laboratoires publics ou privés. 


\title{
La recherche empirique en histoire à l'ère numérique
}

\author{
Nicole DUFOURNAUD
}

\section{Introduction}

L'accès à l'information numérique sur Internet a modifié l'acte empirique de la recherche en histoire dans les archives. La mise à disposition sur Internet de certaines sources primaires - ainsi que des inventaires et des répertoires - facilite le travail à distance des chercheurs et des chercheuses. Quant aux nombreuses sources qui ne seront jamais numérisées, l'apport de la photographie numérique permet de concentrer le travail aux archives sur une courte période quand il fallait, auparavant, plusieurs semaines dans les salles de lecture pour dépouiller et transcrire les documents. Nos pratiques en sont bouleversées et cette évolution fait l'objet de publications dans des revues ou des ouvrages ${ }^{1}$. En revanche, le changement opéré dans le processus d'élaboration des savoirs est peu étudié ${ }^{2}$.

1 Pour un état exhaustif des différents articles de revue et des livres parus récemment en langue française sur le sujet, se référer à GENET (Jean-Philippe) et ZORZI (Andrea) (dir.), Les historiens et l'informatique : un métier à réinventer, Rome, École française de Rome, 2011 ; le numéro spécial de la Revue d'bistoire moderne et contemporaine, 2011/5 n 58-4bis et particulièrement les articles de DELALANDE (Nicolas) et JULIEN (Vincent), «Portrait de l'historien-ne en cyborg » p. 5-29, POTIN (Yann), «Institutions et pratiques d'archives face à la "numérisation". Expériences et malentendus » p. 57-69, RYGIEL (Philippe), «L'enquête historique à l'ère numérique », p. 30-40, de HEIMBURGER (Franziska) et RUIZ (Émilien), «Faire de l'histoire à l'ère numérique : retours d'expériences» p. 70-89. Lire GRANDI (Elisa) et RUIZ (Émilien), «Ce que le numérique fait à l'histoire. Entretien avec Claire Lemercier ", Diacronie. Studi Di Storia Contemporanea 2, $\mathrm{n}^{\circ} 10$, 2012 ;

2 Notons cependant les publications de LAMASSÉ (Stéphane) et RYGIEL (Philippe), "Nouvelles frontières de l'historien », Revue Sciences/Lettres [En Ligne], $\mathrm{n}^{\circ}$ 2, 2014 ; CELLIER (Jacques) et COCAUD (Martine), Le traitement des données en histoire et sciences sociales. Méthodes et outils, Rennes, PUR, 2012 ; DUFOURNAUD (Nicole) et FEKETE (Jean-Daniel), «Utilisation de TEI comme support méthodologique au dépouillement de sources manuscrites. Application aux lettres 
Chercheuse en histoire moderne et utilisatrice d'outils informatiques innovants permettant l'exploration de documents informatisés ${ }^{3}$, je m'interroge sur les méthodes, sur le travail collaboratif et sur le partage des données pour revisiter les sources récemment numérisées par les archivistes. Comment la manipulation des outils informatiques permetelle l'exploitation de documents numérisés ? Comment aller plus loin dans l'exploration et la navigation des données en nombre croissant ? En quoi ces outils - logiciels et langages - modifient non seulement les pratiques des chercheurs et chercheuses en histoire mais surtout apportent du sens à nos textes et nous aident à penser ? Finalement, en quoi le numérique est à la source d'un changement des modes de pensée et d'écriture, passant de la linéarité d'un récit d'autorité à une structuration rendant l'analyse traçable et vérifiable? Cette dernière question résume l'enjeu du numérique appliqué à l'histoire, en même temps qu'elle pourrait apporter des éléments d'explication à une sorte de réticence des chercheurs et des chercheuses en histoire à collaborer avec d'autres disciplines comme l'informatique laquelle a la propriété de rendre beaucoup plus visible un processus intellectuel. Répondre à ces questions, c'est aussi démontrer en quoi la nouveauté principale est le flux du travail, en anglais workflow, entre les Archives et leurs usagers. Pour illustrer mon propos, je décrirai une méthode de travail pour une recherche empirique en histoire, méthode en cours d'expérimentation dans le cadre d'un projet européen, le projet Cendari ${ }^{4}$.

\section{Le temps des humanités numériques ${ }^{5}: 1^{\prime}$ accès à l'information numérique}

Jusqu'aux années 2000, l'enquête historique exigeait une fréquentation assidue et prolongée des chercheurs et des chercheuses dans les différents services d'archives ${ }^{6}$. Le choix des sources s'opérait souvent

de rémission du XVIe siècle dans le duché de Bretagne », L'informatique dans l'enseignement de l'bistoire et la formation des historiens. Pratiques en formation, Paris, L'Harmattan, 2001 ; DUFOURNAUD (Nicole), «Des humanités aux données. Méthodes en histoire à l'ère du numérique. Témoignage d'une e-historienne ", Les cabiers du numérique, $10, \mathrm{n}^{\circ}$ 3, 2014, p. 73-88.

3 Voir mes travaux universitaires sur mon site http://nicole.dufournaud.net

4 Pour en savoir plus, voir http://www.cendari.eu/

5 Allusion au titre de l'ouvrage paru récemment LE DEUFF (Olivier) (dir.), Le temps des humanités digitales. La mutation des sciences humaines et sociales, col. Société de la connaissance, FYP éditions, 2014. Cet ouvrage explique les origines des humanités numériques et ses évolutions. J'utilise le terme français « numérique » et laisse celui de « digital » à mes collègues suisses francophones qui ont gardé le terme anglais.

6 La disparition récente de l'historien Alain Dewerpe a fait l'objet de plusieurs articles en sa mémoire qui rappellent sa revendication de «l'immersion 
dans la salle de lecture avec l'aide des agents des archives voire même grâce au bouche à oreille entre chercheurs et amateurs. Puis, le dépouillement était réalisé soit à l'aide d'une grille établie dès le début du projet, soit par des transcriptions partielles ${ }^{7}$. Finalement, ces dépouillements comportaient bien des erreurs, humainement explicables, qui expliquaient les retours multiples dans ces mêmes lieux d'archives afin de compléter les notes prises trop rapidement et sans la réflexion nécessaire à un dépouillement de qualité, surtout en début de projet. L'usage de la photocopie était limité à la fois pour des raisons économiques et pour des raisons de fragilité des documents comme les parchemins.

Les nouvelles technologies informatiques ont modifié récemment les pratiques en recherche historique ${ }^{8}$.

\section{L'image numérique et l'ordinateur personnel}

L'évolution majeure est l'utilisation de l'image numérique: celle professionnelle réalisée par les Archives ${ }^{9}$ et celle privée recueillie grâce à l'appareil photographique numérique personnel.

Finies les photocopies souvent de mauvaise qualité et leur coût prohibitif ! La rapidité du geste et la simplicité des appareils, et le contrôle immédiat du résultat permettent d'accélérer le travail dans les salles de lecture. En revanche, ultérieurement, une tâche fastidieuse de téléchargement des images, puis de classement et de nommage des fichiers, incombe à l'usager des archives sans qu'aucun outil informatique, à ma connaissance, ne puisse l'aider.

Dès les années 2000, dans les salles de lecture, les ordinateurs portables firent leur apparition: l'utilisation des traitements de textes, tableurs et messagerie devint le quotidien des historiens. Plus récemment, la gestion de la bibliographie avec des logiciels comme Zotero simplifia la vie du chercheur ou de la chercheuse.

Les pratiques se modifièrent également en raison de la mise en ligne des instruments de travail et des premières sources comme les registres

empirique » dans les archives; voir la lettre de l'EHESS $\mathrm{n}^{\circ} 83$, http://lettre.ehess.fr/index.php?9014

7 Trop rarement des transcriptions exhaustives.

8 Ce sujet a été traité récemment lors de deux journées d'études organisées la première le 21 janvier 2015, par les Archives nationales et l'université Paris 8, intitulée «A double fonds, archives des femmes»; le 24 janvier 2015, par l'association Mnemosyne intitulée «Dans les coulisses des archives : où sont les femmes ?", http://www.mnemosyne.asso.fr/mnemosyne/dans-les-coulisses-desarchives-ou-sont-les-femmes/

9 Je n'aborde pas ce sujet qui n'est pas mon propos. 
paroissiaux ${ }^{10}$. En conséquence, les salles de lecture se vidèrent comme si la collation d'informations sur Internet suffisait à satisfaire les besoins de la recherche en histoire ${ }^{11}$.

\section{L'accès à l'information sur le Web : un nouveau vocabulaire à comprendre}

Avec la généralisation des micro-ordinateurs dans les années 1990 et l'utilisation du Web, puis des techniques de numérisation, les archivistes se sont emparés des nouvelles technologies ${ }^{12}$. Situés en amont du travail de la recherche, ces professionnels s'emploient à nous offrir une masse importante de documents numérisés et d'instruments de recherche informatisés.

Un problème majeur se pose alors, celui de la non-culture informatique des historiens (même si l'on constate l'émergence de cours spécialisés et d'outils en ligne) et la méconnaissance des nouveaux usages numériques des archivistes. Auparavant, l'usager des archives devait commencer son travail par la lecture des répertoires et des inventaires dont les préfaces précisaient comment les archives avaient été constituées et classées. Aujourd'hui, il nous faut également comprendre comment les sources sont numérisées, pourquoi certaines ne le seront jamais et feront l'objet d'une simple description, mais surtout comment le système de classement a évolué avec l'informatique.

En pleine révolution numérique, les usagers des archives - et des bibliothèques - sont confrontés à un nouveau vocabulaire : référentiel, autorité, descripteur, vedette! Si un référentiel est une description des normes, la vedette est la rubrique principale dans laquelle sont données toutes les références se rapportant à un nom donné. Il s'agit donc de choisir la référence vedette et de décliner toutes les autres appellations : une recherche effectuée sur les différentes appellations doit aboutir à l'identité vedette. Ainsi dans le domaine des bibliothèques, la BNF utilise un fichier d'autorités, soit une «vedette » avec du contenu qui forme une notice : c'est une «liste d'entrée autorité personne »" Quant au site des Archives de France, l'historien peut y trouver des informations de ce

10 Je n'aborde pas ici la question des archives numériques natives qui ne concernent que les historiens du temps présent.

11 Lire le dossier spécial, PAQUET (Fabien), «Les historiens ont-ils abandonné les archives ? ", L'Histoire, $\mathrm{n}^{\circ} 410$, avril 2015, p. 9-15.

12 Voir l'article de Yann POTIN qui explique en quoi les avancées pionnières ont peut-être étaient un inconvénient pour les archives POTIN (Yann), Ibid.

13 A la BNF, je constate deux listes d'entrées autorité: «autorités BNF» et «RAMEAU » avec des types d'autorités : par exemple «PEP» pour les personnes, «TIC» pour les titres et « ORG» pour les organisations. Je renvoie le lecteur vers le site de la BNF http://rameau.bnf.fr 
type : «Nomina est un schéma $\mathrm{xml}$ spécifique qui permet de décrire toutes les données nominatives possibles ${ }^{14}$. Téléchargeable, ce fichier en format $\mathrm{xsd}^{15}$ peut paraittre complètement abscons au chercheur sans culture informatique ${ }^{16}$. La mutation des métiers des archivistes et des bibliothécaires a précédé celle du métier des chercheurs en histoire. Je reconnais bien volontiers les dangers de la classification qui ne datent pas de l'ère informatique : verrouillage de connaissances qui sont fluctuantes comme les changements d'identités, non-gestion de l'incertitude et de l'approximation, prééminence d'une institution sur une autre pour la diffusion des listes d' " autorité », etc. Si toute classification est réductrice par nature, les indexations et les typologies reviennent en force et nous n'y échappons pas. En fait, les capacités énormes de nos ordinateurs autorisent une exploitation différente des données grâce à des listes qui doivent être dressées automatiquement à partir des textes en collaboration entre les différents spécialistes afin de ne pas commettre d'erreurs ou d'omission.

Ensemble, nous devons veiller à ce que la numérisation des sources, leur catalogage, les normes et leur description soient le reflet de la réalité des données archivistiques.

\section{Collaboration et partage des données}

Le travail considérable de numérisation en cours actuellement dans les archives permet aux historiens de revisiter les sources primaires bien plus nombreuses aujourd'hui à notre portée. Non seulement la distance entre les archives françaises et l'usager est réduite à une connexion internet, mais également avec toutes les archives au monde. Cependant nos cerveaux humains à eux seuls ne sont pas en mesure d'étudier toute cette masse de données: nous devons nous faire aider d'une part par les ordinateurs aux capacités bien plus importantes et d'autre part en acceptant le travail collaboratif et le partage des données. Concrètement, je propose ici le récit d'une collaboration à plusieurs niveaux entre un amateur généalogiste, des historiens, des archivistes et des informaticiens.

Depuis les années 1990, Jacques Rouziou, généalogiste amateur, recueille informatiquement des données aux Archives départementales de Loire-

14_http://www.archivesdefrance.culture.gouv.fr/ressources/bases-dedonnees/ Une traduction possible serait: Nomina est un schéma spécifique en langage de balisage informatique XML (Extensible Markup Language). XML est une norme ISO qui sert à décrire des documents structurés.

15 Ce fichier définit les balises XML valide pour ce projet.

16 Parmi le nouveau vocabulaire, citons également «taxinomie», « ontologie », «folksonomie ». 
Atlantique. Il accepte de les partager d'une part avec des chercheurs en histoire, des étudiant-e-s, et d'autres généalogistes ; de surcroît il remet une copie de sa base de données aux Archives. A partir de nos dépouillements personnels et des documents remis par Jacques Rouziou, Bernard Michon, maitre de conférence en histoire moderne de l'Université de Nantes, et moi-même avons présenté une étude lors d'un colloque organisé par l'Université de Bordeaux : l'intervention portait sur le sujet très en vogue des réseaux sociaux ${ }^{17}$. Afin de réaliser cette intervention, nous avons sollicité la collaboration de Pascal Cristofoli, ingénieur de recherche de l'Ecole des Hautes Etudes en Sciences Sociales (EHESS), et de Benjamin Bach, post-doctorant en informatique à MSRINRIA Joint Centre, tous deux spécialistes des réseaux sociaux. A partir de nos données collationnées depuis quinze années et de celles remises par Jacques Rouziou, nous avons réussi collectivement à construire des réseaux sociaux spatiaux temporels dynamiques ${ }^{18}$.

La participation de chaque protagoniste à un tel projet oblige les détenteurs des données à les remettre. Sans partage, aucune collaboration possible ne peut être envisagée.

\section{Du matériau archivistique à l'analyse historique}

En offrant au plus grand nombre, chercheur ou amateur les matériaux archivistiques qui permettent d'élaborer les récits historiques, les services d'archives enclenchent une nouvelle dynamique et bousculent les modèles : les chercheurs ne sont plus les seuls à accéder aux documents rares qui, scannés, peuvent aujourd'hui être scrutés à l'autre bout de la planète.

Si l'apport des nouvelles technologies oblige les chercheurs et les chercheuses à réfléchir de nouveau sur leurs pratiques, en revanche le processus dans l'enquête historique ne s'est guère renouvelé malgré une modification possible dans la chaine de production des savoirs, du matériau archivistique à la prise de notes pour finalement rédiger un récit de synthèse des analyses qui en découlent. Finalement, il s'agit de comprendre comment le récit final d'un historien se construit, quelle en

17 DUFOURNAUD (Nicole) et MICHON (Bernard) avec la collaboration de Benjamin Bach et Pascal Cristofoli, «L'analyse des réseaux, une aide à penser : réflexions sur les stratégies économique et sociale de Marie Boucher, marchande à Nantes au XVIIe siècle», dans Actes du colloque "Femmes et réseaux dans les sociétés modernes et contemporaines. Réalités et représentations » 16-17 octobre 2014, PU Bordeaux, Bordeaux, à paraître.

18 L'exploration de nos nombreuses données a été rendu possible grâce à l'utilisation d'un outil expérimental, Jigsaw de l'université Georgia Tech. 
est la chaîne de production.

Dans un précédent projet pionnier des années 1990 avec les Archives nationales, le problème du workflow avait déjà été identifié ${ }^{19}$. L'apport majeur de ce projet avait été la résolution des entités nommées grâce à une manipulation intuitive des données que je vais m'employer à vous décrire dans la partie suivante.

À partir de ces premiers résultats, et grâce à l'évolution informatique de ces dernières années ${ }^{20}$, je décris ici une méthode de travail.

\section{Le projet Cendari : une plate-forme en cours de déploiement}

Un des objectifs du projet Cendari ${ }^{21}$ est de construire une plate-forme de travail en ligne. Elle est hébergée par l'infrastructure européenne $\operatorname{Dariah}^{22}$. Cette plate-forme permet de restituer le processus de l'élaboration des savoirs ce qui nécessite la mise en relation des documents scannés, des transcriptions et de la prise de note en lien avec la description des documents d'archives, toutes ces données pouvant être partagées dans le cadre d'un projet. Concrètement, cette plate-forme est conçue pour la saisie collaborative sur Internet et comprend un ensemble de logiciels, formant comme une boite à outils pour l'historien. Parmi ces outils, des logiciels de visualisation et d'exploration des données permettent de préparer la phase ultime du travail de l'historien : le récit.

La construction de cette plate-forme implique de répondre à la question «Comment travaillent l'historien et l'historienne ? " pour mettre en place le flux des tâches que je liste ici, tel un scénario :

錞téléchargement des photos scannées par l'usager ou par les services des archives grâce à Internet, ou récupération d'images,

錞saisie ou copié/collé de la description archivistique des documents, ou encore liaison avec un pointeur sur les sites des services d'archives,

鍗lecture du document image par image,

錞transcription des textes,

19 FEKETE (Jean-Daniel) et OGILVIE (Denise), «Le projet et la plate-forme "millefeuille" recherches et outils informatiques Pour de nouveaux usages des Almanachs ", Bibliothèque de l'École des chartes, 166, no. 1 (2008), p. 89-98.

20 Par exemple, autour des technologies du web sémantique, des langages Python, Java et de nouveaux moteurs de recherche comme Elastic search.

21 Le projet Cendari réunit des archivistes, des informaticiens et des historiens de plusieurs pays européens.

22 https://www.dariah.eu/ 
錞prise de notes,

錞détection et surlignage d'une appellation (nom de personne complet ou incomplet, d'une date, d'un événement, d'un lieu),

錞résolution des entités nommées.

Pour la réalisation de cette dernière étape, la plate-forme propose de choisir un type d'entités : lieu, nom de personne, événement, etc. Au fur et à mesure de cette tâche, des index s'auto-génèrent: ils se présentent sous forme de listes d'occurrences qui renvoient directement aux différents textes surlignés qu'ils soient des documents transcrits ou des prises de notes. La dernière étape est constituée par l'association automatique d'un identifiant à chaque entité par la liaison des occurrences qui apparaissent identiques: phase de mention de l'identification (prénom et nom par exemple), recherche de l'occurrence par un référent s'il existe, création ou sélection, résolution éventuelle d'une ambiguïté par une identité unique à trouver si l'existence d'une homonymie dans les référentiels est constatée. Par exemple, les occurrences dans le texte telles que le «dauphin Henri de France » et le roi «Henri II » n'auront qu'un seul identifiant. Les entités peuvent faire l'objet d'un développement directement dans leur fiche.

L'exploration des données s'effectue également grâce à des outils de visualisation qui permettent de voir pour mieux comprendre, d'émettre des hypothèses ou de les confirmer/infirmer, bref, une véritable aide visuelle à l'analyse textuelle ${ }^{23}$. A l'ère de la massification des textes numérisés, le problème du grand nombre des données à notre disposition semble résolu, en tout cas à un niveau individuel.

Ce système permet une navigation aisée entre le résultat de l'exploitation des données, les listes d'index, et les textes qui sont analysés. Grâce à la production automatique de tables d'analyses qui deviennent des instruments de travail, les multiples index croisés permettent un travail d'écriture facilité. La plate-forme permet ainsi de suivre le processus de l'élaboration des savoirs et de le rendre explicite : il est alors possible de vérifier et de tracer la donnée, et en cas d'erreur qui se propage, de savoir d'où elle vient. Grâce à la traçabilité et tout en gardant le contexte, l'analyse se trouve étayée par un raisonnement dont on peut remonter la

23 Lorsqu'on a des données et qu'on cherche des questions intéressantes, on doit explorer en utilisant nos capacités visuelles pour détecter des phénomènes inattendus. Spécialité ancienne, la visualisation est une aide à l'analyse ; voir les travaux de Jacques Bertin, La sémiologie graphique, Paris, EHESS, 1999 (rééd.). Les travaux de Jacques Bertin sont poursuivis actuellement par Jean-Daniel Fekete et son équipe Aviz de INRIA spécialistes de la visualisation d'informations http://aviz.fr. 
provenance. L'exploitation historique en est simplifiée et corroborée ; les analyses sont vérifiables et réutilisables, les erreurs d'interprétation sont limitées. La recherche en histoire devient reproductible ${ }^{24}$.

Du côté des services d'archives, de simples requêtes sur Internet sur des plates-formes devraient permettre la consultation et le téléchargement des données comme des documents photographiés, des notes, des références bibliographiques qui serviront aux usagers des Archives comme aux archivistes eux-mêmes. Une plate-forme comme celle de Cendari offre la possibilité de demander par une requête quelles sont les ressources qui référencent les url des services d'archives. Une seule obligation est requise : sur la plate-forme, le groupe de chercheurs devra rendre publiques ses données à la fin du projet, partiellement ou totalement. L'idée de l'exclusivité des sources a vécu. En tant que service public, cette symétrie nouvelle donne aux Archives une nouvelle dimension: la récupération automatique des résultats d'une recherche empirique leur donnera le matériau pour imaginer quels autres services apporter au public.

\section{Conclusion}

La numérisation massive des sources aux archives mais également des ouvrages permet de mieux tracer l'information - du document informatif au texte final - car le lecteur dispose de l'image des sources ce qui lui permet de pouvoir vérifier les données; les outils informatiques permettent également de maintenir la chaîne de raisonnement avec les données en « cliquant» sur l'ordinateur.

Dans la conclusion de son article, Yann Potin annonçait la grande innovation en cours de la recherche en histoire: "la cueillette numérique » des documents grâce à la photographie opérée par les

24 Cette problématique a fait l'objet d'une table ronde aux Rendez-vous de Blois: Quelle place pour le numérique dans la recherche sur les archives? le 12 octobre 2013, Table ronde organisée avec le concours de Laurent ROMARY, directeur de recherche à Inria et co-directeur de l'infrastructure de recherche européenne DARIAH, coanimée avec Anna BOHN maître de conférences, Freie universität Berlin, Frédéric CLAVERT, docteur en histoire contemporaine, ingénieur de recherche, Frédérique JOANiC-SETA chef du service Pôles associés/Gallica à la BnF, Maud Medves, responsable éditoriale, inria- Cendari. La table ronde proposait un tour d'horizon des transformations induites par l'introduction de données et méthodes numériques dans la recherche à partir de fonds d'archives. Voir http://www.rdvhistoire.com/-L-actualite $\% \mathrm{CC} \% 81$-de-la-recherche-html 
usagers des archives qui autorisent la mutualisation des données ${ }^{25}$. Dans un précédent article, j'abordais l'évolution du droit d'auteur en cours : «Les auteurs sont dessaisis de leur édition au bénéfice des internautes qui dynamiquement récupèrent les données, les interprètent, les réanalysent et les rediffusen $t^{26} »$. Dans les archives, le problème est identique. Il me semble que le chercheur, est non seulement l'auteur du récit final, mais également celui qui manipule avec son équipe les données mises à disposition par les archivistes. Il peut récupérer facilement des images scannées ou photographiées par lui-même mais également par d'autres, professionnels ou non, qui déposent en ligne ou pas leur « cueillette numérique ». A ce titre, les chercheurs qui ont parfois bénéficié du travail bénévole d'amateurs comme les généalogistes, sont voués à remettre leur travail intermédiaire sur l'objet - le document ainsi que leurs transcriptions et leurs notes. Cette nouvelle publication est bien plus visible sur Internet et est d'ailleurs une forme de protection pour les différents protagonistes des projets: en conséquence, la réutilisation de leurs données s'en trouve sécurisée puisque traçable.

Les Archives sont déjà prêtes à entrer dans la chaîne automatique de production des savoirs: elles n'attendent que le retour des données «cueillies » et interprétées pour les mettre de nouveau à disposition. Un défi que nous devons relever ensemble. Les archivistes nous précèdent dans ce grand bouleversement. Leur travail est essentiel car les documents d'archives nous inspirent et guident nos recherches.

Nicole DUFOURNAUD

Docteure en histoire, membre associée du LaDéHis à l'EHESS

nicole.dufournaud@,laposte.net

La recherche empirique en histoire à l'ère numérique

Les modes de lecture et les outils de communication et de visualisation changent les pratiques du travail en histoire, mais la chaîne de production reste quant à elle sans grande modification. Pourtant, les récents outils informatiques autorisent le passage de méthode singulière à celle généralisable au bénéfice de la recherche empirique historique. La mutualisation des matériaux et la collaboration interdisciplinaire donnent une dynamique à des projets. Si l'accès à l'information dépend en premier lieu du travail de numérisation opéré par les archivistes, en revanche peu de données récoltées et analysées sont remises en retour aux Archives. Pourtant, un défi est en cours : les plates-formes collaboratives sur le Web changeront les relations entre les services des Archives et leurs usagers.

25 POTIN (Yann), Ibid.

26 DUFOURNAUD (Nicole), «Des humanités aux données... », Ibid. 